\section{El registro internacional de ensayos clínicos}

\author{
Luis Gabriel Cuervo, 1 \\ América Valdés ${ }^{2} y$ \\ María Luisa Clark ${ }^{3}$
}

En años recientes, una serie de escándalos particularmente notorios ha menoscabado la confianza pública en la investigación clínica y sus resultados. Han salido a la luz casos concretos de manipulación de datos en los registros de ensayos clínicos y de modificación de protocolos de investigación sin justificación o sin dejar constancia, así como de otras prácticas reprobables (1). Una reciente revisión sistemática de la literatura reveló, por ejemplo, que los resultados de ensayos con ciertos medicamentos antipsicóticos solían favorecer a la fuente de financiamiento del estudio (2). El impacto adverso de tales incidentes ha perjudicado a toda la comunidad investigadora, no solo a sus responsables directos. También se ha censurado el acceso parcial, a veces deliberado, a muchos resultados de investigaciones financiadas con dineros públicos. Como resultado, la comunidad científica tiene que adoptar medidas para restaurar la confianza general en la investigación médica y evitar que se produzcan sesgos y tergiversaciones en la generación y transmisión de sus resultados. El registro de todo ensayo clínico en una base de datos que esté al alcance del público es una medida que se viene proponiendo desde hace varios años para contrarrestar estas tendencias.

A fines del siglo pasado se crearon diversos registros públicos para ensayos clínicos. Destacan algunas iniciativas: en los Estados Unidos se creó un registro financiado con dineros públicos (www.clinicaltrials.gov); en Europa se estableció un metarregistro (www.controlled-trials.com) con el respaldo del Consejo de Investigación Médica (MRC) y del Programa de Investigación y Desarrollo del Sistema Nacional de Salud (NHS) del Reino Unido (3), y la Colaboración Cochrane también asumió un papel pionero con su Cochrane Central Register of Controlled Trials (CENTRAL). En julio de 2004 los Institutos de Investigación Sanitaria de Canadá (Canadian Institutes of Health Research, CIHR) pidieron que todos sus estudios se registraran en www.controlled-trials.com y convocaron una reunión en Ottawa donde se originó la llamada Declaración de Ottawa, cuya primera parte, traducida al castellano, se ha incluido como primicia en este número de la Revista Panamericana de Salud Publica/Pan American Journal of Public Health (RPSP/PAJPH) (4) (la versión original (5) se encuentra en el sitio web del grupo de Ottawa, <http://ottawagroup.ohri.ca/>). La Declaración, de carácter internacional, pide el registro temprano y detallado de todos los ensayos clínicos, de las enmiendas de sus protocolos, y de todos sus resultados. Dicha Declaración fue instrumental, a su vez, para el desarrollo de la Plataforma Internacional de Registro de Ensayos Clínicos (ICTRP), que la Organización Mundial de la Salud (OMS) gestó con el aval de la Asamblea Mundial de la Salud (6) y que se describirá en mayor detalle en párrafos subsiguientes. La Plataforma también ha sido respaldada por una declaración del Comité Internacional de Directores de Revistas Médicas (ICMJE) (7) y por la Federación Internacional de la Industria del Medicamento (IFPMA, por International Federation of Pharmaceutical Manufacturers and Associations) (8).

No obstante los consabidos beneficios de registrar todos los ensayos clínicos y de ofrecer acceso libre a ellos, algunos argumentan que poner al alcance del público esta información podría menoscabar la competitividad comercial de las compañías patrocinadoras y la propiedad intelectual de los investigadores. Sin embargo, estos riesgos pesan menos que los beneficios para el público y ninguno de los argumentos ha sido convincente. La investigación es un proceso complejo; los competidores suelen conocer de antemano los datos que otros van a registrar y sería ingenuo pensar que alguien pudiera apropiarse de una investigación ajena con la escueta información incluida en el registro. 


\section{El contexto general}

Las comunicaciones modernas y los métodos estadísticos permiten resumir y sintetizar los resultados de las investigaciones, acumularlos, crear bases de datos donde almacenarlos y rastrearlos, y analizarlos en conjunto. Ello, a su vez, ha generado la expectativa de que las prácticas y políticas de salud se basen siempre en los datos científicos (evidence) más válidos, fiables y actualizados. Pero, ¿en qué repositorios están esos datos científicos, qué garantiza su fiabilidad y quién tiene acceso a ellos?

Durante decenios, los resultados de la investigación en materia de salud se han publicado en revistas periódicas especializadas que aplican la revisión por pares como medida de control de calidad. Sin embargo, se sabe que esta práctica adolece de muchas carencias. Aunque la pertinencia y calidad metodológica de un estudio determinan, en teoría, si se publica o no, en la realidad influyen muchos otros elementos, tales como lo apetecible que el artículo pueda resultar para determinados lectores; la novedad del contenido; la calidad de la redacción; el idioma de publicación; la prominencia de los autores y sus instituciones; el respaldo financiero que tenga el estudio; lo polémicos que sean sus resultados y su posible impacto comercial, etc. Estudios pertinentes e importantes pueden quedar relegados a causa de incentivos o presiones perversas, sobre todo del temor a que perjudiquen económicamente a las compañías patrocinadoras o a que generen culpas o una mala imagen de las prácticas vigentes. Otras veces los propios directores de las revistas abrigan sesgos, como indica la rara publicación de resultados sin significación estadística, es decir, de resultados que no confirman la eficacia de la intervención estudiada. Y como las bases de datos bibliográficas de acceso libre, tales como PubMed o Lilacs, cubren esencialmente la investigación publicada e indizan solamente una fracción selecta de todas las revistas en el mundo, muchos resultados pasan inadvertidos. ¿Cómo incorporar y aprovechar entonces esos otros resultados que no se dan a conocer ni se indizan pero que también forman parte del acervo de datos probatorios (evidence) necesarios para respaldar las decisiones y políticas en materia de salud?

Indizar en una sola base de datos pública mundial todos los protocolos de ensayos clínicos aprobados por los comités de ética es una de las propuestas de mayor acogida. La conveniencia de esta medida es obvia. Los centenares de registros que están dispersos ahora se integrarían en una especie de metarregistro o "almacén" mundial con un único portal de acceso. Este es el fundamento teórico de la Plataforma Internacional de Registro de Ensayos Clínicos propugnada por la OMS, que se describirá más adelante. Pero primero examinemos más de cerca algunos de los posibles beneficios de un registro universal de esta naturaleza.

\section{Los beneficios del registro internacional de ensayos clínicos}

Un registro de alcance mundial proporcionaría acceso libre a todas las pruebas científicas acumuladas hasta cierto momento y permitiría saber qué datos hay que actualizar, integrar o sintetizar, qué vacíos del conocimiento existen aún, y qué fuentes contienen todos los datos en un área en particular. Asimismo, permitiría examinar las virtudes y carencias de los estudios realizados y de la metodología científica aplicada en ellos. El registro expondría a la luz pública datos nunca divulgados y enriquecería los metaanálisis y otros estudios basados en datos secundarios.

Otra ventaja radicaría en poder evaluar la eficacia e inocuidad de diferentes intervenciones, idear investigaciones en áreas donde faltan conocimientos, u observar tendencias generales en el campo de la investigación. El registro tam- 
bién serviría para comparar protocolos en detalle: las observaciones de los comités de ética, el tamaño de la muestra final, la congruencia del estudio adelantado con el protocolo propuesto, y demás. Sería un recurso adicional para impulsar la transparencia en la diseminación y divulgación de la producción científica y la participación del público en los ensayos clínicos. El registro permitiría, además, identificar a expertos en áreas particulares del conocimiento; establecer indicadores de producción; establecer las necesidades en materia de investigación, y observar de cerca el desarrollo de la ciencia.

\section{La Plataforma Internacional de Registro de Ensayos Clínicos de la OMS}

La Plataforma Internacional de Registro de Ensayos Clínicos ideada por la OMS es un plan para consolidar todos los registros de ensayos clínicos que existen en el mundo. La Plataforma establece normas sobre la manera de registrar los ensayos y en particular sobre la información mínima que todo investigador o ente patrocinador tiene que aportar a la hora de registrar su estudio, a fin de garantizar que la información consignada en los registros sea uniforme, completa, veraz y precisa. La OMS también contempla la creación de un portal único de entrada a una extensa red de "registros primarios" (Network of Member Registries), que deberán tener su aval para garantizar la adhesión a las normas establecidas. El trabajo en red con estos registros primarios lo coordinará la OMS en un esfuerzo por lograr transparencia, regular la investigación sanitaria, defender los principios científicos y éticos que deben guiar la investigación, y proteger el bien público.

Para maximizar la utilidad del portal, la OMS aboga por asignarle a cada estudio registrado una clave numérica única - el Número Universal de Referencia para Ensayos Clínicos o UTRN (por Universal Trial Reference Number) - , que con singularidad referenciará estudios incluidos en más de un registro. La obtención del conjunto de datos mínimos necesarios para poder asignar el UTRN es una de las responsabilidades de los registros primarios.

Mediante la Plataforma, la OMS también aspira a alcanzar otros objetivos: promover la asignación equitativa de recursos para la investigación; evitar la duplicación de investigaciones; propiciar la realización de estudios pertinentes y afines con las necesidades; impulsar las investigaciones que más ayudarían a mejorar la salud de los pueblos; facilitar el seguimiento de normas éticas, y restaurar la confianza del público en la investigación de salud.

La OMS presentó formalmente el proyecto de la Plataforma en el año 2005 y organizó consultas técnicas en torno a las características y estándares de los registros, el UTRN y la necesidad de un portal mundial que permitiera efectuar búsquedas eficientes de los registros. Estableció que deberían registrarse los ensayos clínicos prospectivos con personas sometidas deliberadamente a una o más intervenciones por el investigador y se acordó que los registros tuvieran como mínimo 20 campos de información útil y esencial que permitiera detectar duplicaciones. Son los siguientes: la fecha de incorporación del estudio en el registro primario; cualquier número de identificación del ensayo además del UTRN; las fuentes de financiamiento principales y secundarias; las personas a quienes dirigirse; el nombre del investigador principal; el título público del estudio; el título científico del estudio; la verificación de aprobación del comité de ética; la enfermedad o trastorno estudiado; las intervenciones evaluadas; los criterios de inclusión y de exclusión; el diseño metodológico; la fecha de incorporación del primer participante; el tamaño muestral necesario; las fechas del reclutamiento; y las variables de interés primarias y secundarias. Se espera que el lanzamiento del portal y del UTRN se anuncien más adelante en el transcurso de 2006. 


\section{La posición de la Organización Panamericana de la Salud}

Para la Organización Panamericana de la Salud (OPS) es grato testificar que la OMS ha tenido la iniciativa de idear la Plataforma Internacional de Registro de Ensayos Clínicos y los mecanismos que permitirán ponerla en práctica. Cabe señalar, sin embargo, que la voluntad política no basta para que el registro resulte provechoso. La OMS tendrá que garantizar que el portal sea accesible y que la información incorporada sea útil, completa y fiable. Deberá, asimismo, facilitar los medios para dar a conocer la Plataforma y cerciorarse de que se utilice de forma apropiada. En la Región de las Américas estos esfuerzos serán encabezados por la Organización Panamericana de la Salud.

La OPS adoptará estrategias para propiciar la adhesión a la Plataforma y fomentar su uso adecuado trabajando con investigadores, comités de ética y ciencia, autoridades reguladoras, agentes financiadores, casas editoriales, organizaciones científicas, organizaciones representantes de pacientes, y muchas otras entidades para crear un ambiente que favorezca la adhesión y observación de los estándares establecidos por la Plataforma, y para que en la Región se cuente con suficientes registros primarios. La OPS también sentará el ejemplo instaurando procedimientos y mecanismos estandarizados para monitorear la investigación que fomenta y apoya, adoptando el registro sistemático de los ensayos clínicos y promoviendo la divulgación pública de los resultados de la investigación.

Desde el punto de vista de la OPS, la investigación de salud es esencial para fortalecer la asistencia sanitaria. Por consiguiente, es esencial que los mejores datos probatorios sean libremente accesibles a todos en formatos estandarizados, que se usen en la síntesis del conocimiento y que informen las políticas y acciones de salud. La OPS considera que el registro de los ensayos clínicos es importante para fomentar la transparencia y aprovechar óptimamente los resultados de la investigación. En consecuencia, está invitando a toda parte vinculada con la investigación de salud a contribuir al desarrollo, fortalecimiento y utilización de la Plataforma y aspira, en particular, a promover el registro, la diseminación y el buen uso de los resultados de la investigación financiada con recursos públicos.

Para impulsar la Plataforma la OPS aplicará los siguientes mecanismos:

- Invitará a las autoridades sanitarias, organismos nacionales de ciencia y tecnología, y otros entes reguladores en los Estados Miembros a adoptar el registro de ensayos clínicos como política general. Asimismo, supervisará la adhesión a esta política en las Américas y promoverá medidas encaminadas a lograr que el portal de la OMS refleje la producción científica de la Región. Se procurará que las autoridades de salud normalicen el registro fidedigno y completo de los ensayos clínicos, en particular de los 20 componentes mínimos esenciales establecidos por la OMS, y que estos se reflejen en el portal de dicho organismo.

- Facilitará la identificación de registros primarios avalados por la OMS, sobre todo de los más accesibles y pertinentes para la Región.

- Abogará ante los comités de ética para que promuevan el registro de los ensayos clínicos en bases primarias. El Comité de Ética de la OPS sentará el ejemplo pidiendo que los ensayos clínicos avalados por la Organización se registren después de ser aprobados por los comités de ética locales, y abogará por el uso del UTRN.

- Promoverá entre las organizaciones patrocinadoras y financiadoras de investigaciones el registro obligatorio de los ensayos clínicos (y el UTRN) como condición para el financiamiento y el reclutamiento de los participantes. 
- Solicitará a los editores de revistas médicas que exijan el registro de todo ensayo clínico en bases de datos que alimenten el portal de la OMS.

- Fomentará el uso del UTRN para la presentación de ensayos clínicos en eventos científicos.

- Propiciará la normalización jurídica necesaria para que en cada país de la Región se registren los protocolos de todos los ensayos clínicos.

Es responsabilidad de la OMS procurar que los resultados de la investigación científica se usen para mejorar la salud y que se apliquen instrumentos y métodos que permitan evaluar la calidad y utilidad de las intervenciones de salud. Esto implica normalizar los procesos y mecanismos orientados a lograr que la investigación sanitaria sea ética, metodológicamente rigurosa, y útil (9). Mediante su apoyo al registro internacional de los ensayos clínicos, la OPS colaborará con otros impulsores y beneficiarios de la investigación y con la sociedad civil para conseguir que el portal de la OMS sea una iniciativa meritoria y sostenible, fortalezca los sistemas de salud y restaure la confianza en la investigación.

\section{La función de las revistas biomédicas}

Las revistas biomédicas y sus directores están en una posición idónea para fomentar la transparencia y las prácticas éticas en el campo de la investigación. Estimuladas por la Plataforma de la OMS, muchas revistas médicas han impuesto el registro como requisito para la publicación de estudios. El registro obligatorio se aplica a ensayos clínicos con seres humanos, con o sin grupo testigo o aleatorización, sin importar el tipo de intervención realizada o la fase en que se encuentre el ensayo. Por "intervención" se entiende cualquier medicamento, tratamiento quirúrgico o psiquiátrico, dispositivo o prótesis, técnica de modificación conductual, asesoramiento u otra medida con que se busca un resultado sanitario en particular. Por lo pronto no es preciso registrar estudios epidemiológicos o ecológicos que evalúan retrospectivamente o prospectivamente los efectos sanitarios de exposiciones naturales, sin una intervención deliberada.

En consonancia con la política de la OMS, la redacción de la $R P S P / P A J P H$ respalda de lleno el registro obligatorio de todo ensayo clínico como requisito para su publicación. Asimismo, su profundo interés en fomentar la ética y la transparencia en el ámbito de la investigación sanitaria suscitó la publicación en el número de julio de 2003 de "Normas para la publicación de investigaciones clínicas patrocinadas por la industria farmacéutica" (10) y de "La ética de la publicación médica: entre la academia, el estado y la industria" (11) y explica la publicación en el número actual de la versión en castellano de la Declaración de Ottawa y de un trabajo de Reveiz et al. sobre el registro de ensayos clínicos en América Latina $(4,12)$. Esperamos que los lectores de la $R P S P / P A J P H$ encuentren en estos documentos motivos suficientes y valederos para persuadirse de respetar y propugnar las normas internacionales delineadas por los Institutos de Investigación Sanitaria del Canadá en su Declaración de Ottawa y por la Plataforma de la Organización Mundial de la Salud.

\section{REFERENCIAS}

1. Chan AW, Altman DG. Identifying outcome reporting bias in randomised trials on PubMed: review of publications and survey of authors. BMJ. 2005;330(7494):753. [PMID 15681569].

2. Marshall E. Antidepressants and children. Buried data can be hazardous to a company's health. Science. 2004; 304(5677):1576-7. [PMID 15192185]

3. Tonks A. A clinical trials register for Europe. BMJ. 2002;325(7376):1314-5. [PMID 12468459]

4. Krleža-Jerić K, Chan AW, Dickersin K, Sim I, Grimshaw J, Gluud C, en nombre del grupo de Ottawa. Principios del registro internacional de protocolos y resultados de ensayos clí- 
nicos a base de intervenciones de salud en seres humanos: Declaración de Ottawa (parte 1). Rev Panam Salud Publica. 2006;19(6):413-6.

5. Krleža-Jerić K, Chan AW, Dickersin K, Sim I, Grimshaw J, Gluud C, for the Ottawa Group. Principles for international registration of protocol information and results from human trials of health interventions: Ottawa statement (part 1). BMJ. 2005;330(7497):956-8.[PMID 15845980].

6. World Health Organization. International Clinical Trials Registry Platform. Disponible en: URL:http:/ / www.who.int/ictrp/en/ Citado el 31 de mayo de 2006.

7. International Committee of Medical Journal Editors. Uniform requirements for manuscripts submitted to biomedical journals: writing and editing for biomedical publication. Disponible en: http:/ /www.icmje.org/ Citado el 2 de junio de 2006.

8. Federación Internacional de la Industria del Medicamento (IFPMA). Postura conjunta sobre la revelación de información de ensayos clínicos mediante registros y bases de datos. Disponible en http:/ / www.ifpma.org/clinicaltrials.html. Citado el 31 de mayo de 2006.

9. Organización Mundial de la Salud. Función y responsabilidades de la OMS en las investigaciones sanitarias. Informe de la Secretaría. 117ª Reunión: Punto 4.12 del orden del día provisional. EB117/14. 16-1-2006. Disponible en http://www.who.int/gb/ebwha/pdf files/EB117/B117_14-sp.pdf. Citado el 1 de junio de 2006.

10. Normas para la publicación de investigaciones clínicas patrocinadas por la industria farmacéutica. Rev Panam Salud Publica. 2003;14(1):62-6. [PMID 12952617].

11. Lolas F. La ética de la publicación médica: entre la academia, el estado y la industria. Rev Panam Salud Publica. 2003;14(1):1-2. [PMID 12952600].

12. Reveiz L, Delgado MB, Urrutia G, Ortiz Z, Dieguez MG, Martí-Carvajal A, et al. The Latin American Ongoing Clinical Trial Register (LATINREC). Rev Panam Salud Publica. 2006; 19(6):417-21 